\title{
Dicipivirus (family Picornaviridae) in wild Northern white-breasted hedgehog (Erinaceus roumanicus)
}

\author{
Gábor Reuter ${ }^{1,2} \cdot$ Ákos Boros ${ }^{1,2} \cdot$ Gábor Földvári $^{3} \cdot$ Sándor Szekeres $^{3} \cdot$ Róbert Mátics $^{4,5} \cdot$ Beatrix \\ Kapusinszky $^{6} \cdot$ Eric Delwart $^{6,7} \cdot$ Péter Pankovics ${ }^{1,2}$
}

$\square$ Gábor Reuter reuter.gabor@gmail.com

1 Regional Laboratory of Virology, National Reference Laboratory of Gastroenteric Viruses, ÁNTSZ Regional Institute of State Public Health Service, Pécs, Hungary

2 Department of Medical Microbiology and Immunology, Medical Center, University of Pécs, Szigeti út 12, Pécs 7624, Hungary

3 Department of Parasitology and Zoology, University of Veterinary Medicine, Budapest, Hungary

4 Hungarian Nature Research Society (HuNaReS), Ajka, Hungary

5 Department of Nature Conservation, Kaposvár University, Kaposvár, Hungary

6 Blood Systems Research Institute, San Francisco, CA, USA

7 University of California, San Francisco, CA, USA 


\begin{abstract}
Using random amplification and high-throughput sequencing technology a novel picornavirus with dicistronic genome organization and genetically related to canine picodicistrovirus (genus Dicipivirus, family Picornaviridae) was identified and characterized in Northern white-breasted hedgehogs. Hedgehog dicipivirus (hedgehog/H14/2015/ HUN, MF188967) was detected in 15 (75\%) of 20 faecal specimens by RT-PCR with high viral loads (up to $8.2 \times 10^{8}$ genomic copies $/ \mathrm{ml}$ faeces). Hedgehog dicipivirus RNA was also identified in blood, ear skin, abdominal muscle and liver tissues. While the general dicistronic genome organization of hedgehog/H14/2015/HUN is similar to canine picodicistrovirus (5'UTR-P1-IGR-P2/P3-3UTR) there are some unique genome characteristics within the untranslated regions, especially in the functional IRES elements. This study reports the putative second member of the genus Dicipivirus, in a novel host species.
\end{abstract}


Picornaviruses are genetically diverse group of viruses (taxonomically classified within the family Picornaviridae) that have been found in various vertebrate host species. At pre- sent, the family is divided into 35 genera and 80 species - as of May 2017 [1]. In general, picornaviruses have $\sim 6.7-9.9 \mathrm{~kb}$ long +ssRNA genome and traditionally encode a single polyprotein transcribed from a single open reading frame (ORF) flanked by a 5' and 3' untranslated region (UTR) and a poly(A)-tail [2]. The P1 region, encoding the capsid proteins, is located at the 5' end of the picornavirus genome. In 2012, a novel picornavirus - called canine picodicistrovirus 1 ( CaPdV-1) - with a naturally occurring, unique dicistronic genome was described for the first time [3]. Until now, this canine picodicistrovirus is the sole representative of species Cadicivirus A, genus Dicipivirus (Picornaviridae), viruses with a picornavirus-like genome organization (gene order). Importantly, however, the P1 (capsid proteins) and P2/P3 (non-structural proteins) regions of canine picodicistrovirus 1 are encoded in two ORFs separated by a functionally active intergenic region (IGR) containing an internal ribosomal entry site (IRES), a phenomenon present in members of the family Dicistroviridae. Canine picodicistroviruses were originally detected from faecal samples of dogs collected in 2007/2009, in Hong Kong [3]. Until now, there have been no further reports or additional identification/confirmation of dicipivirus in dogs or any other animal hosts.

The Northern white-breasted hedgehog (Erinaceus roumanicus) is a medium-sized mammal in the genus Erinaceus, family Erinaceidae (Mammalia: Eulipotyphla). It can be found in Central Europe, Balkan Peninsula, Adriatic Islands, Ukraine and Russia (as far as the Ob River in Siberia). The Northern white-breasted hedgehog inhabits farmland, parks and gardens in rural and urban areas, scrubby habitats at the edge of forests, and shrubby vegetation [4]. They feed on earthworms, insects (larvae, pupae and imagoes as well), snails and slugs, small vertebrates (amphibians, lizards and occasionally young rodents), chicks and eggs of birds and even some berries and fruits [5]. Like its congener the European hedgehog (E. europaeus), it is more abundant in artificial than in natural habitats [4].

Using random amplification and high-throughput sequencing technology a novel picornavirus with dicistronic genome organization and genetically related to canine picodicistrovirus was identified and characterized in Northern white-breasted hedgehog. This study reports the second putative member of the genus Dicipivirus identified in a novel host species.

A total of 20 faecal specimens were collected from North- ern white-breasted hedgehog (Erinaceus roumanicus). Eight faecal samples (H1, H2, H4, H9-H11, H13 and H14) were collected from carcasses of road-hit hedgehogs collected from April to June in 2015, from urban environments, mainly $(\mathrm{N}=7)$ from the streets of the capital Budapest, and one from the suburb Szentendre, Hungary. Twelve faecal samples (MR1-MR9 and MR11-MR13) were collected from wild-living hedgehogs (collected from 
April to July in 2015) from natural wild areas near the town of Pécs, Baranya County, Southwest Hungary. Except H10 and H11 which were juvenile animals (<1-year-old) the other animals were adults ( $>$ 1-year-old) based on the animal size and - if it was possible to investigate - the skull bone ossification. The distance between the two sampling areas is $170 \mathrm{~km}$. Samples were collected by qualified biologists with valid permissions (the National Inspectorate for Environment, Nature and Water: 4018-4/2015). The collected faecal samples were stored at $-80{ }^{\circ} \mathrm{C}$. A specimen pool containing 3 faecal samples (H9, H13 and H14) collected from 3 Northern white-breasted hedgehogs (from dead animals which were hit by cars whilst on roads) was randomly selected for viral metagenomics analysis. Briefly, the PBS-diluted specimen was passed through a $0.45-\mu \mathrm{m}$ sterile filter (Millipore) and centrifuged at $6,000 \mathrm{Xg}$ for $5 \mathrm{~min}$. Subsequently the filtrate was treated with a mixture of DNases and RNases to digest unprotected nucleic acids [6]. Viral-particle protected nucleic acids were extracted using the QIAamp spin-column technique (Qiagen) and subjected to a viral metagenomic analysis using sequence independent random amplification [7]. A viral cDNA library was constructed by Nextera XT DNA Library Preparation Kit (Illumina) and then the library was sequenced on the HiSeq Illumina plat- form according to the manufacturer's instruction, and also as described previously [6]. The acquired reads were trimmed; de-novo assembled and analyzed using an in-house pipeline [6]. The reads and contigs greater than 100-bp were compared to the GenBank protein database (BLASTx). Virus familylevel categorization of viral metagenomic reads was based on the best BLASTx-scores (E-value $\leq$ $10^{-10}$ ). Sequence specific screening primer pairs (dicipi-F: 5'-TCA ATCCCGGATGGAAGAAAC-3' corresponding to $\mathrm{nt}$ positions 3639-3659 and dicipi-R: 5'-ATAGAAACACGGAACACCGAAA-3' corresponding to nt positions 4002-3981 of the study strain) were designed based on the IGR-IRES sequence contig to identify the picornavirus RNA from the specimen pool. Different sets of specific primers were designed to the metagenomic reads/contigs to obtain the complete viral genome (hedgehog/H14/2015/HUN) and for verification of the metagenomic contigs by primer-walking, 5'/3'RACE and TAIL-PCR methods. PCR-products were sequenced directly and then run on an automated sequencer (ABI Prism 310, Applied Biosystems, Stafford, USA). All faecal specimens ( $\mathrm{N}=$ 20) from Northern white-breasted hedgehogs were tested by RT-PCR using dicipi-F/dicipi-R as primerpairs for screening. In addition, available coagulated blood (H14), ear skin (H1, H4 and H14), abdominal muscle (H4, H13 and H14), spleen (H4 and H14) and liver (H1 and H14) tissues were also tested by RT-PCR using the same dicipi-F/dicipi-R primers. All evolutionary analyses were conducted in MEGA 6.06 [8]. The complete structural (P1) and non-structural (3CD) amino acid sequences of the study strain and representative picornaviruses were aligned by MEGA 6.06 and pre-tested using a best aa model (ML) search. Dendrograms were constructed by the Maximum-likelihood method based on the Poisson 
model with gamma distribution $(+\mathrm{G})$ and invariable sites $(+\mathrm{I})$. Assessing the reliability of the trees bootstrap values were provided and determined with 1000 replicates.

The RNA secondary structure of the 5'UTR and IGR IRES were predicted using Mfold [9] (Zucker 2003) with manual correction in order to locate the analogous structures found in canine picodicistrovirus $[3,10,11]$. Possible poly- protein cleavage sites were predicted from alignments with canine picodicistrovirus (JN819202) polyproteins.

Quantification reactions are based on picornavirus cDNA transcribed using the reverse primer H14R 5'TCCCACAAG ACTGGTCGTTCC-3' (corresponding to nt positions 3739- 3719 of the study strain) and amplified by primers (H14R/ dicipi-F) designed against the IGR region using a real-time PCR assay (LightCycler FastStart DNA Master SYBR Green I, Roche, Mannheim, Germany). For absolute quantification and the generation of a standard curve, a hundred-fold dilution series of a silica-column (Qiagen, Hilden, Germany) purified, and spectrophotometrically quantified, single PCR amplicon of the picornavirus was used. In the qPCR analysis three technical repeats were included.

A specimen pool containing three faecal samples from Northern white-breasted hedgehogs was subjected to viral metagenomics analysis. After de novo assembly of the 23.004.466 total number of reads, 315.105 sequence reads were obtained showing similarity to viruses from this pool. The detected sequences containing more than 50 reads were from viruses taxonomically classifiable within the Picornaviridae $(\mathrm{N}=151.949)$, unclassified viruses $(\mathrm{N}=83.300)$, Tombusviridae $(\mathrm{N}=54.383)$, Virgaviridae $(\mathrm{N}=10.829)$, Microviridae $(\mathrm{N}=8.810)$, Alphaflexiviridae $(\mathrm{N}=2.250)$, Circoviridae $(\mathrm{N}$ $=1.536)$, Permutotetraviridae $(\mathrm{N}=441)$, Luteoviridae $(\mathrm{N}=398)$, Parvoviridae $(\mathrm{N}=370)$, Podoviridae $(\mathrm{N}=225)$, Tymoviridae $(\mathrm{N}=99)$, Carmotetraviridae $(\mathrm{N}=79)$, Iflaviridae $(\mathrm{N}=71)$, and Dicistroviridae $(\mathrm{N}=67)$. The 93.943 reads and the 5 sequence contigs (between 1060 and 6620 nts in length) corresponding to canine picodicistrovirus (JN819202) (genus Dicipivirus, family Picornaviridae) were selected for further analysis. Using the screening primer (dicipi-F/ dicipi-R), 2 (H13 and H14) of the 3 specimens from the sample pool were RT-PCR-positive for the dicipivirus. To characterize the complete picornavirus genome from sample $\mathrm{H} 14$ different sets of specific primers were designed on the basis of the metagenomic sequence reads and amplicons were sequenced directly by Sanger sequencing. The complete genome of the dicipivirus strain hedgehog/ H14/2015/HUN (MF188967) is 8,838 nt long including a 776-nt 5' end, a 588-nt IGR and a 130-nt 3' end excluding the poly(A)-tail (Figure 1). The $\mathrm{G}+\mathrm{C}$ content of the genome is $46.7 \%$. The $\mathrm{P} 1$ region of hedgehog/H14/2015/ HUN encodes a capsid polyprotein of 919 aa (2,760 nt) that is 75aa longer than the corresponding P1 of canine picodicistrovirus (JN819202). Dicipiviruses including hedgehog/H14/2015/HUN do not have a putative L-protein preceding the capsid region. Compared to 
picodicistrovirus (JN819202), respectively.

Phylogenetic analysis based on the complete aa sequences of the P1 and 3CD proteins showed that hedgehog/H14/2015/HUN clustered together with canine picodicistrovirus, viruses classifiable within the genus Dicipivirus (Figure 2).
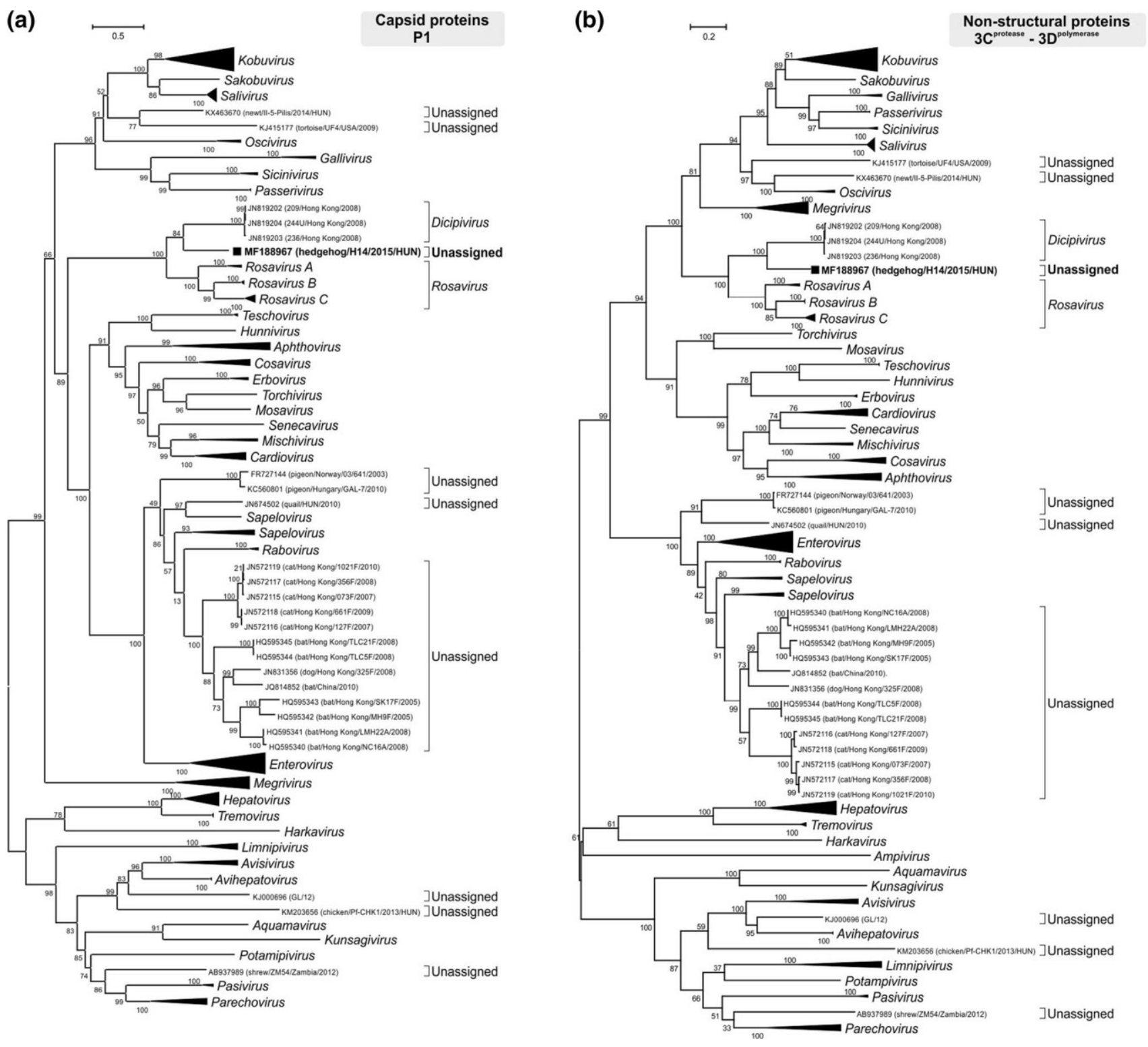

Fig. 2 Phylogenetic analysis of strain hedgehog/H14/2015/HUN (MF188967) (bold letter) and representative picornaviruses based on the complete A) P1 structural and B) 3CD non-structural protein sequences. These evolutionary analyses were conducted in MEGA6 [8], and evolutionary history was inferred using the Maximum-likelihood method based on the Poisson correction model with discrete Gamma distribution $(+\mathrm{G})$, allowing evolutionarily invariable sites $(+\mathrm{I})$. Altogether, 172 sequences were involved in the analysis using all sites; bootstrap values were determined with 1000 replicates. The tree was drawn to scale, with branch lengths representing the number of substitutions per site. 
The 5'UTR of the hedgehog/H14/2015/HUN is 776nt long, which is 206nt shorter than the 5'UTR of canine picodicistrovirus (Figure 1). By sequence comparison the nt identity of the two 5'UTR regions was $38 \%$, but this was higher (57\%) at the 5' end of the 5'UTR (from nt position 647 to 776 of hedgehog/H14/2015/HUN; within the IRES). The canine picodicistrovirus IRES comprises 12 domains (d1-d12) [10]. It diverges structurally from canonical type-I IRESs but contains essential motifs (including GNRA tetraloop motif) in subdomains d10c and d11 (Figure 3) that is homologous to poliovirus $\mathrm{dIVc}$ and $\mathrm{dV}$ and requires the same IRES trans-acting factor, the poly(C)-binding protein 2 (PCBP2) [10, 11]. Structural homology is apparent between IRES elements (d8- d12) of canine picodicistrovirus and the 5'UTR IRES of hedgehog/H14/2015/HUN, such as within the domains d8, d9 and $\mathrm{d} 11$ as well as the pyrimidine(Y)-tract (Figure 3). There are also major differences: the structure of the $\mathrm{d} 10$ of hedgehog/H14/2015/HUN lacks equivalent sequences (including the GNRA tetraloop) found in $\mathrm{d} 10$ of canine picodicistrovirus and is presently unpredictable; it also lacks the d12 domain (Figure 3).

The 588nt long IGR of the hedgehog/H14/2015/HUN is similar in length and shows $64 \%$ nt identity to the IGR of canine picodicistrovirus (Figure 1). Structural homology is apparent between IGR IRES elements (I-VI) of canine picodicistrovirus [3] and the corresponding IGR region of hedgehog/H14/2015/HUN, such as the domains I, II, III, V and the pyrimidine(Y)-tract (Figure 3). However, structural differences are also identifiable in domains IV and VI. The nt sequence and the secondary RNA structure of hedgehog/H14/2015/HUN domain d11 (5'UTR IRES) and domain V (IGR IRES) are highly similar to each other (Figure 3).

The 3'UTR is 130nt long and 299nt shorter than the 3'UTR of canine picodicistrovirus (Figure 1). Similar nt sequence was not found in GenBank. 
(a)5'UTR IRES

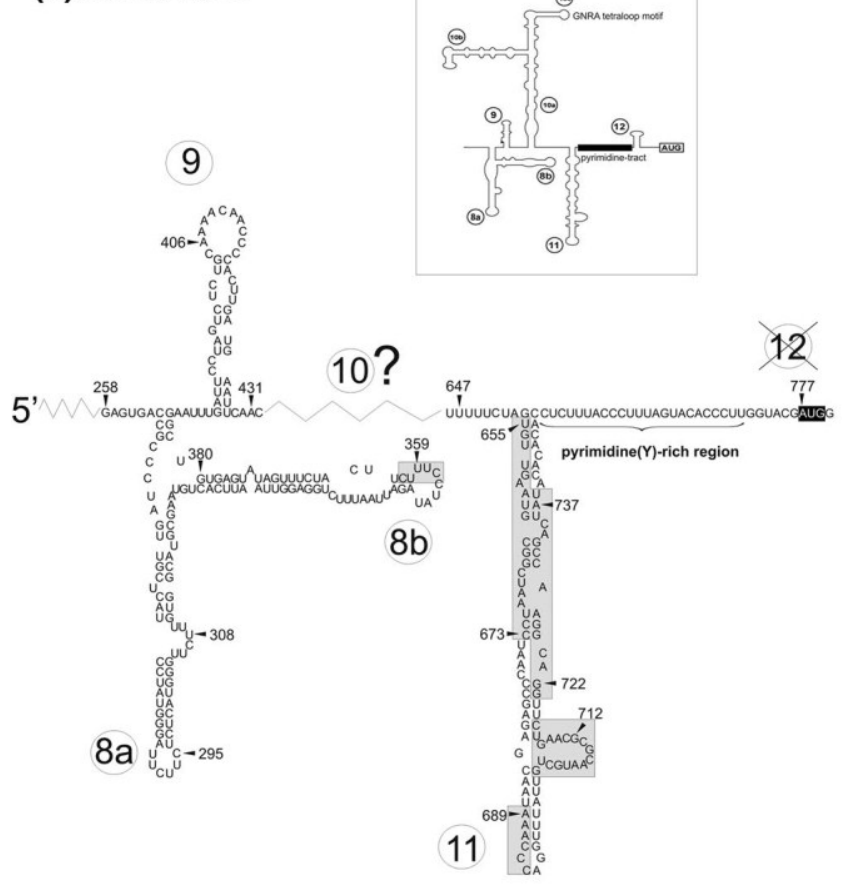

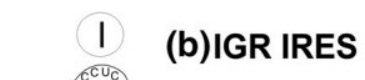

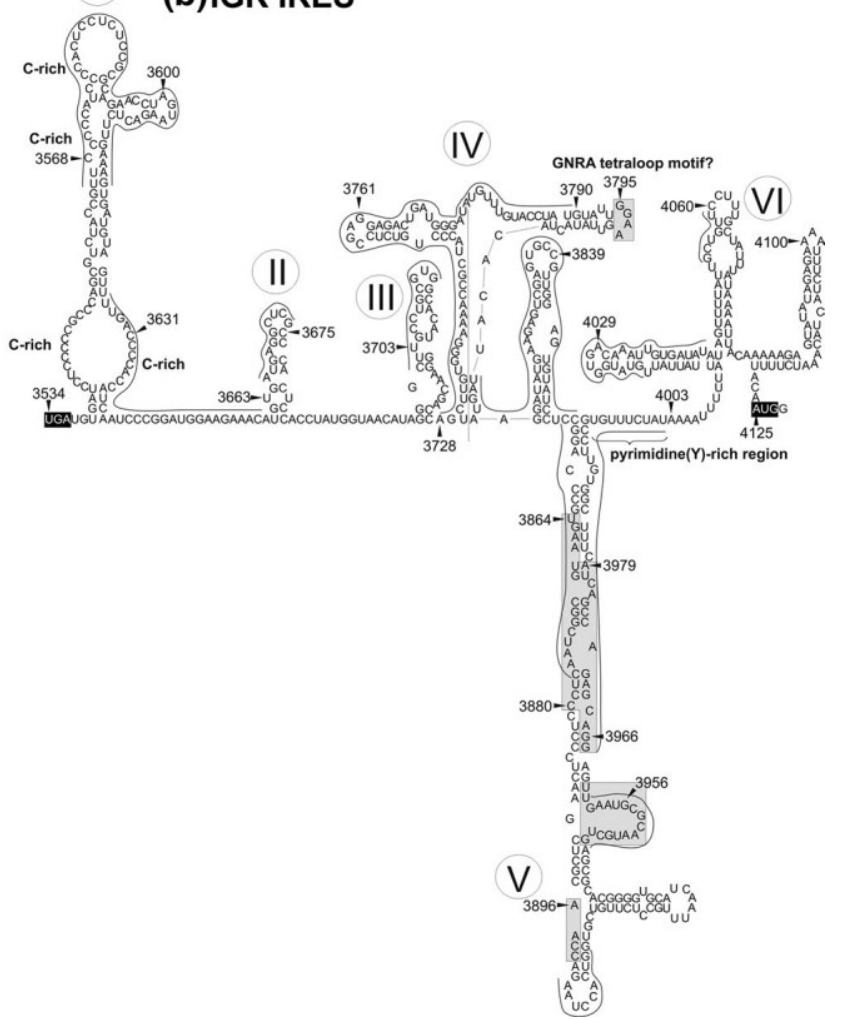

Fig. 3 The predicted secondary RNA structure of (a) the partial 5'UTR IRES and (b) IGR IRES of the dicipivirus strain hedgehog/ H14/2015/HUN (MF188967). The RNA structures, domains and con- served motifs (shaded boxes) have been annotated, as proposed previously [3, 10, 11]. The 5'UTR IRES of hedgehog/H14/2015/HUN domain d10 is unpredictable; it also lacks the $\mathrm{d} 12$ domain. The AUG initiation and the stop codons are indicated by black boxes. The continuous line represents the conserved nucleotides between canine picodicistrovirus (JN819202) and hedgehog/H14/2015/HUN in IGR IRESes.

Using the dicipi-F/dicipi-R screening primer-pairs a total of $15(75 \%)$ of the 20 faecal specimens collected from Northern white-breasted hedgehogs from two geographic regions were RT-PCR-positive for dicipivirus; all were confirmed by direct nucleotide sequencing. Four (50\%) of the 8 and $10(83 \%)$ of the 12 faecal specimens contained dicipivirus originated from urban/suburban (Budapest and Szentendre) and wild natural (Pécs) areas, respectively. The result of the real-time PCR quantification of hedgehog/H14/2015/HUN reveals the presence of $8.2 \times 10^{8}$ genomic copies/ml faeces (SD: $\pm 2.02 \times$ $10^{8}$ ) in sample H14. Available tissues samples collected from dead hedgehogs from urban/suburban (Buda- pest and Szentendre) areas with dicipivirus-positive faecal sample were also tested for dicipivirus by RT-PCR using the dicipi-F/dicipi-R primers. A total of 6 (55\%) of the 11 tissue samples were RT-PCR-positive; all were confirmed as dicipivirus sequences using direct nucleotide sequencing. In detail, dicipivirus RNA was detected in blood (H14), ear skin (H1), abdominal muscle (H4, H13 and H14) and liver (H14) specimens. Based on the amplified 321-bp-long IGR there was 100\% nucleotide sequence identity between the H14 sequence that originated from faeces, muscle, blood and liver. The 
nucleotide sequence identity was $99 \%$ between sequences from different individuals in the same genome region. The results of the real-time PCR quantification of hedgehog/H14/2015/HUN revealed the presence of $1.03 \times 10^{7}, 1.03 \times 10^{5}$ and $7.73 \times 10^{5}$ genomic copies/ml in abdominal muscle, blood and liver tissues from $\mathrm{H} 14$, respectively.

Dicipiviruses are a novel and genetically unique group of viruses, classifiable within the family Picornaviridae. Until now, only the prototype canine picodicistrovirus was known, which was discovered from faecal samples in dogs [3]. The genetic diversity, origin and host species spectrum of this group of viruses remains open to further investigation. This study reports the identification, quantification and complete genome characterization of a second member of this genus in faecal samples and - for the first time - in different tissue samples collected from wild Northern whitebreasted hedgehogs in two geographic areas in Hungary. Using RT-PCR and qPCR this dicipivirus was detected at a high frequency (three fourth of the specimens were RT-PCR-positive) in these animal faeces; a high viral load in the faecal and tissue samples was also determined. The highest number of metagenomic reads related to picornaviruses, by viral metagenomics. Dicipivirus RNA was also identified in blood, ear skin, abdominal muscle and liver tissues. Identical nucleotide sequences were detected in the different specimens collected from the same animal. These results indicate that dicipiviruses are common in the tested hedgehog population and may replicate in this potential host species. Hedgehog/H14/2015/HUN VP1 and 3D proteins have 43\% and 29\% aa sequence divergence when compared to the corresponding proteins of canine picodicistrovirus, respectively. Based on sequence- and phylogenetic analysis hedgehog/H14/2015/HUN represents, taxonomically, a potential novel species within the genus Dicipivirus. While the general dicistronic genome organization of hedgehog/H14/2015/ HUN is similar to canine picodicistrovirus (5'UTR-P1-IGR- P2/P3-3UTR) there are some unique genome characteristics, especially within the untranslated regions and the two functional IRES elements. Similar to the 5'UTR-IRES of canine picodicistrovirus the 5'UTR-IRES of hedgehog/H14/2015/ HUN has a type-I-like IRES but differences are present in sequence length as well as the possible RNA secondary structures of the IRES domains d10 and d12. Domain d10 is not predictable by comparison with $\mathrm{d} 10$ of canine picodicistrovirus while d12 is probably missing in hedgehog/ H14/2015/HUN. Indeed, experimental evidence suggests that domain d12 is not essential for IRES function in canine picodicisitrovirus [10]. Similarly, structural differences were also identified in domains IV and VI of IGR-IRES between these two dicipiviruses.

Exotic pets, including hedgehogs, have become popular in recent years among pet owners, especially in North America [12]. All pets have a flora of microbes, and it should be known which are potentially zoonotic. Hedgehogs pose a risk for a number of potential zoonotic viral infections 
including bunyaviruses (Tahyna virus, CCHF), flavivirus (tick-borne encephalitis) and rabies. In addition, they could impact on susceptible livestock, e.g. through Foot-and- mouth (picorna)virus transmission [12]. A recent study has also reported a novel hepatovirus (family Picornaviridae) in a faecal sample from hedgehog [13]; however, most virological studies target E. europaeus and as a result our current knowledge of viruses in E. roumanicus is limited. In this study, faecal samples collected from E. roumanicus hedgehogs in Hungary potentially contained a minimum of 14 different (bacterial, plant, fungal, insect and mammal) virus families. Interestingly, the picornavirus, and especially the dicipivirus sequence, reads outnumbered all other known viral sequence reads using our viral metagenomics platform. Detection and characterization of further members of the genus Dicipivirus will help researchers to understand the origin, evolution, genetics and host species diversity of picorna and picorna-like viruses with dicistronic genome organizations.

Acknowledgements This work was supported by a Grant from the Hungarian Scientific Research Fund (OTKA/NKFIH K111615), by the Hungarian Nature Research Society and by NHLBI R01-HL105770. P.P., Á.B. and G.F. were supported by the János Bolyai Research Scholarship of the Hungarian Academy of Sciences. The GenBank [/EMBL/ DDBJ] accession number for the study sequence: MF188967.

\section{Compliance with ethical standards}

Funding This study was funded by the Hungarian Scientific Research Fund (OTKA/NKFIH K111615) and by the Hungarian Nature Research Society and NHLBI R01-HL105770.

Conflict of interest The authors declare that they have no conflict of interest.

Ethical approval All applicable international, national, and/or institutional guidelines for the care and use of animals were followed. 


\section{References}

1. Knowles NJ (2017) The picornavirus pages. http://www.picorna- viridae.com/. Accessed 17th May 2017

2. Knowles NJ, Hovi T, Hyypiä T, King AMQ, Lindberg AM, Pal- lansch MA, Palmenberg AC, Simmonds P, Skern T, Stanway G, Yamashita T, Zell R (2012) Picornaviridae. In: King AMQ, Adams MJ, Carstens EB, Lefkowitz EJ (eds) Virus taxonomy:classification and nomenclature of viruses: ninth report of the international committee on taxonomy of viruses. Elsevier, San Diego, pp 855-880

3. Woo PC, Lau SK, Choi GK, Huang Y, Teng JL, Tsoi HW, Tse H, Yeung ML, Chan KH, Jin DY, Yuen KY (2012) Natural occur- rence and characterization of two internal ribosome entry site elements in a novel virus, canine picodicistrovirus, in the picor- navirus-like superfamily. J Virol 86:2797-2808

4. Lapini L (1999) Erinaceus concolor. In: Mitchell-Jones AJ, Amori G, Bogdanowicz W, Kryštufek B, Reijnders PJH, Spitzenberger F, Stubbe M, Thissen JBM, Vohralík V, Zima J (eds) The atlas of European mammals. Academic Press, London, pp 36-37

5. Szekeres S, Majláthová V, Majláth I, Földvári G (2016) Neglected hosts: the role of lacertid lizards and medium-sized mammals in the eco-epidemiology of Lyme borreliosis. In: Braks MAH, van Wieren SE, Takken W, Sprong H (eds) Ecology and prevention of Lyme borreliosis (ecology and control of vectorborne diseases, volume 4). Wageningen Academic Publishers, Wageningen, pp 103-126

6. Phan TG, Vo NP, Boros Á, Pankovics P, Reuter G, Li OT, Wang C, Deng X, Poon LL, Delwart E (2013) The viruses of wild pigeon droppings. PLoS One. 8:e72787

7. Victoria JG, Kapoor A, Li L, Blinkova O, Slikas B, Wang C, Naeem A, Zaidi S, Delwart E (2009) Metagenomic analyses of viruses in stool samples from children with acute flaccid paralysis. J Virol $83: 4642-4651$

8. Tamura K, Stecher G, Peterson D, Filipski A, Kumar S (2013) MEGA6: molecular evolutionary genetics analysis version 6.0. Mol Biol Evol 30:2725-2729

9. Zucker M (2003) Mfold web server for nucleic acid folding and hybridization prediction. Nucleic Acids Res 31:3406-3415

10. Asnani M, Pestova TV, Hellen CUT (2016) Initiation on the diver- gent type I cadicivirus IRES: factor requirements and interactions with the translation apparatus. Nucleic Acids Res 44:3390-3407

11. Asnani M, Pestova TV, Hellen CUT (2016) PCBP2 enables the cadicivirus IRES to exploit the function of a conserved GRNA tetraloop to enhance ribosomal initiation complex formation. Nucleic Acids Res 44:9902-9917

12. Riley PY, Chomel BB (2005) Hedgehog zoonosis. Emerg Infect Dis 11:1-5

13. Drexler JF, Corman VM, Lukashev AN, van den Brand J, Gmyl AP, Brünink S, Rasche A, Seggwi $\beta$ Feng H, Leijten LM, Vallo P, Kuiken T, Dotzauer A, Ulrich RG, Lemon SM, Drosten C (2015) 
Evolutionary origins of hepatitis A virus in small mammals. PNAS 112:15190-15195 